\title{
Active Learning Configuration Interaction for Excited States Calculations of Polycyclic Aromatic Hydrocarbons
}

\author{
WooSeok Jeong $^{\mathrm{a} \dagger}$, Carlo Alberto Gaggioli ${ }^{\mathrm{b} \dagger}$ and Laura Gagliardi ${ }^{\mathrm{b} *}$
}

a) Department of Chemistry, Nanoporous Materials Genome Center, Chemical Theory Center, and Minnesota Supercomputing Institute, University of Minnesota, Minneapolis, Minnesota 55455, United States

b) Department of Chemistry, Pritzker School of Molecular Engineering, James Franck Institute, Chicago Center for Theoretical Chemistry, University of Chicago, Chicago, Illinois 60637, United States

$\dagger$ W.J. and C.A.G. contributed equally to this work

*To whom correspondence should be addressed.

\begin{abstract}
We present the active learning configuration interaction (ALCI) method for multiconfigurational calculations based on large active spaces. ALCI leverages the use of an active learning procedure to find important electronic configurations among the full configuration space generated within an active space. We tested it for the calculation of singlet-singlet excited states of acenes and pyrene, by using different machine learning algorithms. The ALCI method yields excitation energies within $0.2-0.3 \mathrm{eV}$ from those obtained by traditional complete active space configuration interaction (CASCI) calculations (affordable for active spaces up to 16 electrons in 16 orbitals), by including only a small fraction of the CASCI configuration space in the calculations. For larger active spaces (up to 26 electrons in 26 orbitals), not affordable with traditional CI methods, ALCI captures the trends of experimental excitation energies.
\end{abstract}


Overall ALCI provides satisfactory approximations to large active-space wave functions with up to ten orders of magnitude fewer configurations. These ALCI wave functions are promising and affordable starting points for subsequent second order perturbation theory or pair-density functional theory calculations.

\section{Introduction}

Electronic excited states of organic materials play a key role in many applications, such as photovoltaics, ${ }^{1-}$ ${ }^{3}$ light emitting diodes ${ }^{4,5}$ and photochemistry..$^{6-8}$ The computational analysis of excited states of organic materials such as hydrocarbon molecules (e.g., aromatic molecules and polyenes) ${ }^{9}$ and porous organic polymers (e.g., conjugated organic polymers, hyper-crosslinked polymers and covalent organic frameworks) ${ }^{10,11}$ is important to rationalize experimental spectroscopic results and make predictions. In this regard, the most widely used methods for ab initio computations are density functional theory (DFT) and time-dependent DFT (TDDFT). ${ }^{12-15}$ However, a major limitation of DFT is that it may provide inaccurate results for phenomena where strong correlation plays an important role, ${ }^{16}$ such as bond-breaking processes, ${ }^{17,18}$ spin states energetics ${ }^{19-21}$ and excited states energetics. ${ }^{22,23}$ Strong electronic correlation, sometime referred as static correlation, arises when different electronic states are close in energy, whose wave functions can be correctly described as linear combinations of several Slater determinants (SDs) or configuration state functions (CSFs) with a non-negligible contribution. ${ }^{24,25}$ The complete active space selfconsistent field (CASSCF) method ${ }^{26}$ is widely used to generate reference wave functions for strongly correlated systems. In CASSCF, an active space consisting of a given number of orbitals and electrons is chosen, and a full configuration interaction (FCI) is performed within the active space, together with the orbital optimization. Usually, the spin and spatial symmetry of the wave function are specified.

The number of CSFs scales exponentially with the size of the active space. ${ }^{27}$ The maximum number of electrons and orbitals that one can afford in modern computers is about 16 electronic and 16 orbitals for a singlet state calculations, which corresponds to $10^{8}$ to $10^{9} \mathrm{CSFs}$. Few examples of larger active spaces, like, 
for example, 22 electrons in 22 orbitals, using massive parallelization have been reported. ${ }^{28}$ Some approximations to reduce the number of configurations have been developed, including the restricted active space SCF (RASSCF), ${ }^{29}$ the generalized active space SCF $(\mathrm{GASSCF})^{30}$ and the localized active space SCF (LASSCF). ${ }^{31}$ In RASSCF and GASSCF subspaces of electrons and orbitals are chosen, and the maximum number of electronic excitations between subspaces is restricted to the number that the user decides. In LASSCF, the active space is partitioned in multiple active sub-spaces which are localized on spatially separated parts of the molecule. The FCI wave function within each sub-space is obtained independently from the other active sub-spaces, and the total wave function is expressed as a product of these unentangled wave functions. Although these approaches allow the choice of extremely flexible active spaces, they also require an expertise in the choice of subspaces and excitation levels between different subspaces. It is also possible to only optimize the configuration interaction coefficients and not the orbital coefficients, resulting in a complete active space configuration interaction (CASCI) calculation, and the analogous GASCI and LASCI. The CASCI method, however, still involves a FCI calculation within the chosen active space.

To reduce the number of CSFs one can perform a "selected CI (SCI)" calculation, in which many nonimportant configurations are not included in the wave function. In this case the challenge is to identify the important configurations. Recently, SCI methods have been revisited for the computation of properties of strongly correlated systems. ${ }^{32-36}$ SCI methods aim to construct a compact wave function iteratively, including only a small number of determinants or configurations, to approximate the properties of the FCI wave function. One flavor of SCI is to use perturbation theory to select important configurations, like in the configuration interaction using an iterative perturbative selection (CIPSI). ${ }^{37}$ In the adaptive sampling CI approach ${ }^{38}$ the single and double excitations are generated only from configurations with the highest coefficients, and then they are selected using perturbation theory. This method has been recently employed in combination with very large active spaces, up to $(52 \mathrm{e}, 52 \mathrm{o}) .{ }^{39}$ In the heat-bath $\mathrm{CI}(\mathrm{HCI})$ method, an approximation to the full expression of first-order perturbation is used to select configurations. ${ }^{40}$ In Monte Carlo configuration interaction (MCCI) ${ }^{41-43}$ configurations are stochastically chosen and only those with a coefficient higher than a certain threshold are retained in the wave function. 
In recent years, machine learning (ML) has been increasingly used in quantum chemistry, ${ }^{44-49}$ to accelerate coupled cluster calculations, ${ }^{50,51}$ excited state computations ${ }^{52}$ and predict quantum mechanical wave functions, ${ }^{53}$ to only mention a few. In particular, an active learning (AL) approach ${ }^{54}$ has been used to minimize the amount of training data, thus reducing the overall training cost. In AL the performance of a supervised ML model can be maximized with fewer labeled data if the ML model can choose data for the next training step from those learned in previous training steps. AL schemes have been successfully integrated in quantum chemistry, in combination with molecular dynamics ${ }^{55-57}$ and for materials discovery, ${ }^{58}$ especially when unlabeled data (e.g., new atomic configurations or new crystal structures) can be easily generated, while labeling of the data is difficult and time-consuming (e.g., obtaining quantummechanical (QM) properties via ab initio calculations).

Recently, an active ML approach has been used to identify important configurations in SCI ground state calculations of small molecules. In the method called machine learning configuration interaction (MLCI), ${ }^{59}$ an artificial neural network (ANN) regression model has been used to learn on the fly to choose important configurations in an iterative SCI scheme. This method significantly reduces the number of iterations to converge the selection of configurations and requires less time compared to other approaches such as CIPSI and MCCI. MLCI recovered up to $98 \%$ of the FCI correlation energy for some multireference problems, like the computation of the dissociation of $\mathrm{CO}$ and $\mathrm{H}_{2} \mathrm{O}$. In a following paper, MLCI was used to compute potential energy curves for $\mathrm{N}_{2}, \mathrm{CO}$ and $\mathrm{H}_{2} \mathrm{O}$, and the results were of FCI quality. ${ }^{60}$ Another example is an ML-based SCI method called Chembot, which utilizes a support vector machine (SVM) with a Gaussian radial basis function (RBF) kernel. ${ }^{61}$ Unlike the MLCI approach, the Chembot method adopted a SVM model to directly classify important and not important configurations to iteratively construct the wave function. By developing features using charge density matrix and configuration energy, and inclusion of heuristics for better training data selection, Chembot can reach chemical accuracy and near exactness in total energy calculations for $\mathrm{H}_{4}, \mathrm{H}_{2} \mathrm{C}$, and $\mathrm{H}_{2} \mathrm{O}$. Both MLCI and Chembot have focused on the ground state of small molecules. In different approaches, ANNs have been employed to determine the relative weights 
of configurations for computing the ground state of one- and two-dimensional Heisenberg spin chains without repeated SCI calculations, ${ }^{62}$ and reinforcement learning techniques have been tested to calculate the ground state energies for dissociation curves of $\mathrm{CO}, \mathrm{N}_{2}$ and an $\mathrm{H}_{8}$ chain, and larger hydrogen rings up to $\mathrm{H}_{14}{ }^{63}$

Inspired by the MLCI approach, we developed an active ML protocol to find important configurations to perform CASCI calculations with large active spaces. We apply the method to compute the lowest singlet-singlet excitation of several polycyclic aromatic hydrocarbons (PAH) as shown in Figure 1. We focus on linear PAH, going from naphthalene to hexacene, and on pyrene, as an example of a non-linear PAH. These molecules are chosen because it is known that their first singlet excited state acquires significant multireference character as the size increases, resulting in interesting electronic structure and properties as promising organic optoelectronic materials. ${ }^{64-66}$

The paper is structured as follows: in section 2 we describe the active learning CI (ALCI) protocol, the protocol of the quantum mechanical calculations and the machine learning methodologies. In section 3 we describe the ALCI results; in section 4 we offer our conclusions and a perspective about the use of the ALCI method.
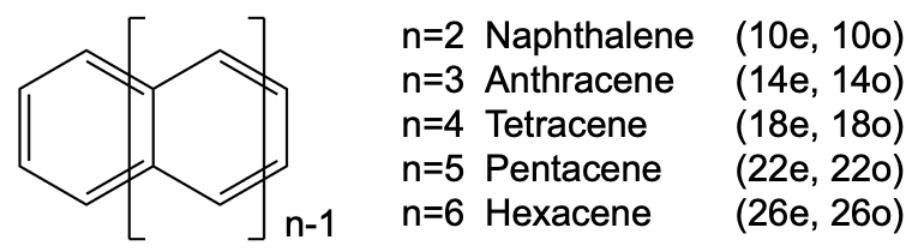<smiles></smiles>

Pyrene (16e, 16o)

Figure 1. Molecular structure of polyacenes and pyrene. $\mathrm{n}$ is the number of fused benzene rings. $(n, N)$ is the active space size where $n$ is the number of active electrons (herein, the number of $\pi$ electrons) and $N$ is the number of active orbitals (i.e., the number of $\pi$ bonding and $\pi^{*}$ antibonding orbitals). 


\section{Active Learning Configuration Interaction Protocol}

In order to find important configurations within the full configuration space spanned by the active space, we devised an active learning CI protocol, which uses an iterative workflow controlled via an in-house Python code integrated with an external general CI program, the GENCI program ${ }^{67}$ in the general atomic and molecular electronic structure system (GAMESS) package, ${ }^{68,69}$ as shown in Figure 2. The GENCI program performs multiconfigurational calculations with arbitrary user-specified configurations (i.e., SCI calculations). A pool-based active learning scheme was adopted to separate configurations into a labeled and unlabeled data pool. An oracle (i.e., the labeler which is the GAMESS software) is used to label configurations into unimportant (label "0") and important (label "1"), and then the configurations and their labels are saved in a labeled data pool. During the iterative procedure, a label for a configuration in the labeled data pool can be changed depending on the outcome of the SCI calculation. Unlabeled data are produced by generating excited configurations from only important configurations, which is the same approach as used in the adaptive sampling CI method, ${ }^{38}$ and then added to the unlabeled data pool after removing any duplicates of the original important configurations. A subset of data from the labeled data pool is utilized for training an ML model, and then the trained ML model is used to predict if the generated excited configurations in the unlabeled data pool are important or not. Queries (i.e., unlabeled configurations to be labeled by the oracle) are selected from the unlabeled data pool based on ML predictions. The selected queries are labeled with the use of GAMESS, i.e., we verify whether the ML predictions are accurate or not with the use of the oracle. We repeat this cycle to update data pools and generate excited configurations iteratively, until the excitation energy is converged within a user-defined energy difference $(0.01 \mathrm{eV}$ is used in this work). 


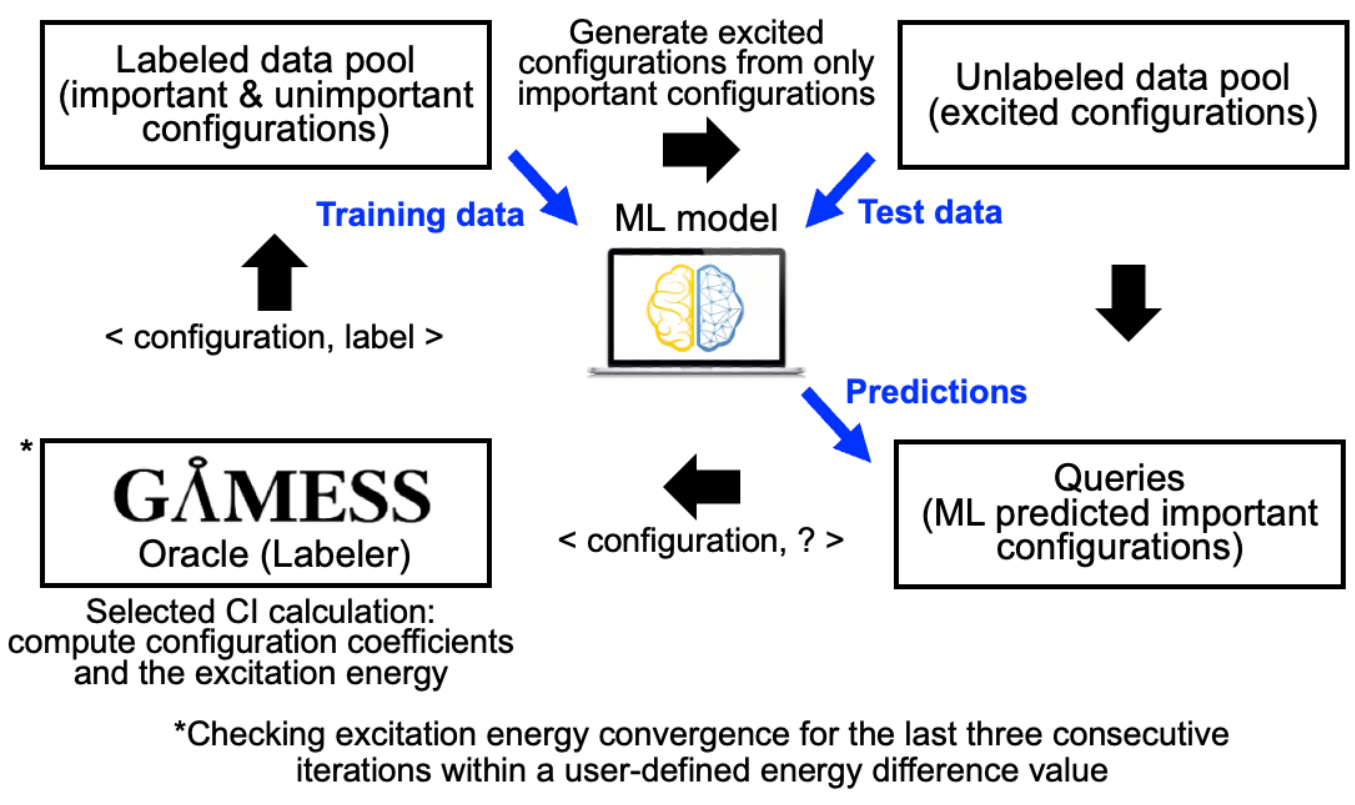

Figure 2. Active learning scheme for finding important configurations in iterative selected CI calculations.

The detailed workflow of the active learning CI protocol is shown in Figure 3. The protocol is divided into three steps: initialization, iteration, and termination. In the initialization step one obtains the initial data to start the iterative process. For a given molecule, the geometry optimization is performed using the Gaussian09 software, ${ }^{70}$ employing the M06-L $\mathrm{L}^{71}$ density functional and the def2-TZVP basis set. ${ }^{72,73}$ An ultrafine grid is used for numerical integration. We then start the ALCI protocol using the GAMESS (US) software ${ }^{68,69}$ with the cc-pVDZ basis $\operatorname{set}^{74}$ and an active space that contains the $\pi$ and $\pi^{*}$ orbitals. The procedure is set up to use Hartree-Fock (HF) guess orbitals, but different guesses can in principle be used. A restricted active space CI calculation including only single and double excitations from HF (referred to as $\operatorname{RASCI}(\mathrm{n}=2))$ is performed.

The second step consists of the iterative scheme to identify only important configurations within the FCI space corresponding to a given active space. Important and unimportant configurations from the SCI calculation at the previous iteration (or the RASCI $(n=2)$ for the first iteration cycle) are identified based on a coefficient threshold (herein, 0.01 or 0.005 ). If the configuration coefficient is higher than or equal to 
the threshold, then the configuration is labeled as important. The labeled configurations are used to update the labeled data pool. To find further important configurations, excited configurations are generated up to a user-specified maximum level of excitations only from the important configurations previously identified, and then added to the unlabeled data pool. Next, training and test data sets are constructed from the labeled and unlabeled data pools, respectively. Since the majority of the configurations will be unimportant, the training data set will most likely be highly imbalanced if naïve random sampling is adopted. To prevent this, an undersampling scheme is adopted to sample the same number of unimportant configurations randomly compared to the important configurations. 


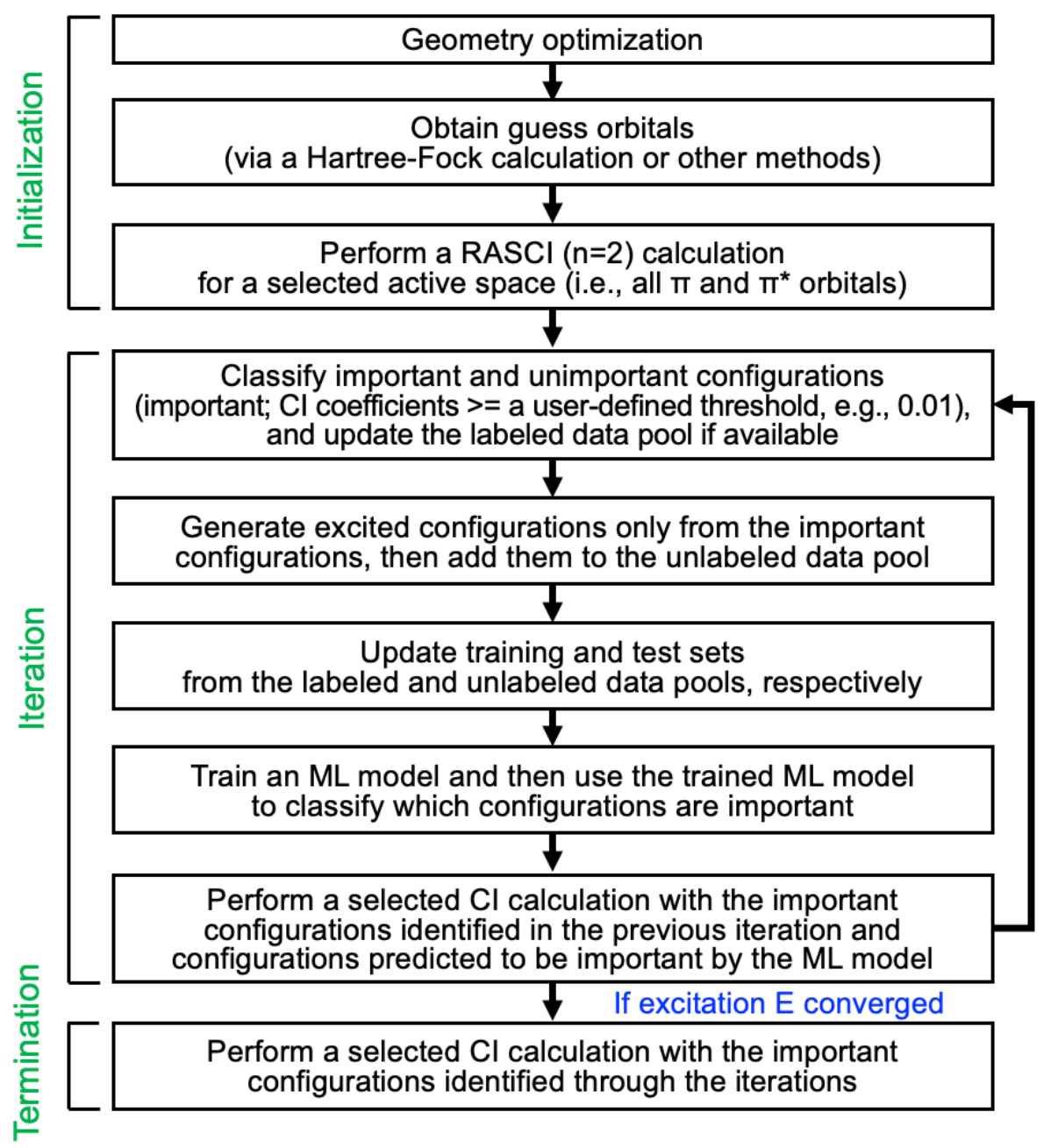

Figure 3. Workflow of the active learning configuration interaction (ALCI) protocol.

A binary classification machine learning model is adopted to predict whether a given configuration is important or not. For featurization of a configuration, an array that contains the active orbitals occupation numbers (either 2, 1 or 0 ) divided by 2 is used (see Supporting Information, section S1). The length of the array is therefore equal to the number of orbitals in the active space. Noteworthy, we do not have to consider the electron spin in the featurization scheme, as the SCI calculation input requires only the specification of the occupation numbers (without spin) for each configuration, and the GAMESS program then generates all possible spin combinations arising from the specified configurations. Furthermore, no symmetry of the wave function is currently used in the calculations. However, in principle symmetry could be included. Six 
different ML algorithms are employed to develop a binary classifier: Kernel ridge regression-based classifier (KRC), ${ }^{75}$ k-nearest neighbors $(\mathrm{KNN}),{ }^{76}$ Gaussian processes $(\mathrm{GP}),{ }^{77}$ random forest $(\mathrm{RF}),{ }^{78}$ gradient boosting decision tree (eXtreme Gradient Boosting, XGBoost) ${ }^{79}$ and artificial neural networks (ANNs). ${ }^{80}$ KNN, GP, and RF classifiers are used as implemented in the scikit-learn package ${ }^{81}$ while KRC is adopted by modifying the kernel ridge regressor in the scikit-learn no classifier model uses the kernel ridge regression in the package. The open source gradient boosting Python library XGboost ${ }^{79}$ is used for the gradient boosting decision tree algorithm. ANN models with three hidden layers are adopted using the skorch library ${ }^{82}$ with PyTorch ${ }^{83}$ as the backend. For each iteration, hyperparameter tuning is newly performed to maximize the ML model performance (herein, the F1 score is used as a scoring method) using the HyperOpt, ${ }^{84}$ a Bayesian optimization Python library, with 10-fold cross-validation (CV) except for ANNs where 5-CV is used considering expensive training cost. Further details regarding the ML model training and hyperparameter tuning are available in the Supporting Information.

The trained ML model is then employed to classify provisional important configurations from the test set that should be labeled using the GENCI program in the GAMESS package. An SCI calculation including all the important configurations plus some (or all) important configurations predicted by the ML model (i.e., queries) is performed to update important configurations and compute the excitation energy. To reduce the computational cost, the number of the ML-predicted important configurations added for the SCI calculation is limited by a user-specified number. If the specified number is smaller than the number of training data in the previous step, the number of added ML predicted configurations is set to the specified value, otherwise it is set to the number of training data. If the computed excitation energy is not converged, the second step is repeated until the excitation energy is converged. The calculation is converged when the excitation energy changes by less than $0.01 \mathrm{eV}$ for three consecutive iterations. In the termination step, one additional SCI calculation is performed to obtain the final excitation energy value with all the important configurations previously identified. 


\section{Results and Discussion}

\subsection{Sensitivity to Iteration Parameters}

The following iteration parameters were tested to check the convergence of the ALCI calculations (in parenthesis the baseline values for each parameter are reported): i) maximum number of iterations for each SCI calculation (3, details are available in the Supporting Information (SI), section S3.1), ii) maximum sampling number of queries (2000, see SI, section S3.2), iii) maximum level of excitations for each iteration (1), iv) query sampling method (using class probability for sampling priority), v) CI coefficient threshold for important configurations (0.01). Three independent calculations following the above protocol were conducted considering the stochastic nature of the ML model. For this reason, in the following, we will report the average number of iterations and average time of these three independent calculations. Naphthalene, anthracene, and tetracene were used as test systems, and a classifier based on the kernel ridge regression was employed to speed up the sensitivity test.

As mentioned in the Section 2, unlabeled data are created as excited configurations from the important configurations identified via an SCI calculation. In this step one has to specify the maximum level of excitation (e.g., singles, doubles, triples, or a higher excitations) from the reference configurations like in multireference CI (MRCI) methods. ${ }^{85}$ Generating higher excitations (for example quadruple and quintuple excitations) from a large number of reference configurations is time and memory intensive, as in the MRCI methods. ${ }^{86}$ Therefore, we have tested two ways: Generate (1) only single excitations, and (2) single and double excitations. Note that our configuration generation method does not limit the CI expansion by truncating it to a specific excitation level like truncated CI methods, so one automatically generates higher level excitations, as products of lower-level excitations, like in coupled cluster theory. As shown in Figure 4, generating both single and double excitations has no noticeable advantages over generating only single excitations. For naphthalene, including up to double excitations converges faster than including only single excitations (i.e., average 7.3 vs. 9.0 iterations). However, for larger systems such as anthracene and tetracene, the higher excitations resulted in a similar or slower convergence (on average, 13.3 and 16.3 
iterations, using single and double excitations, and 11.3 and 15.0 iterations using only excitations for anthracene and tetracene, respectively) though the converged excitation energies are similar. This suggests that single excitations from important configurations are sufficient to generate unlabeled configurations in the ALCI protocol.
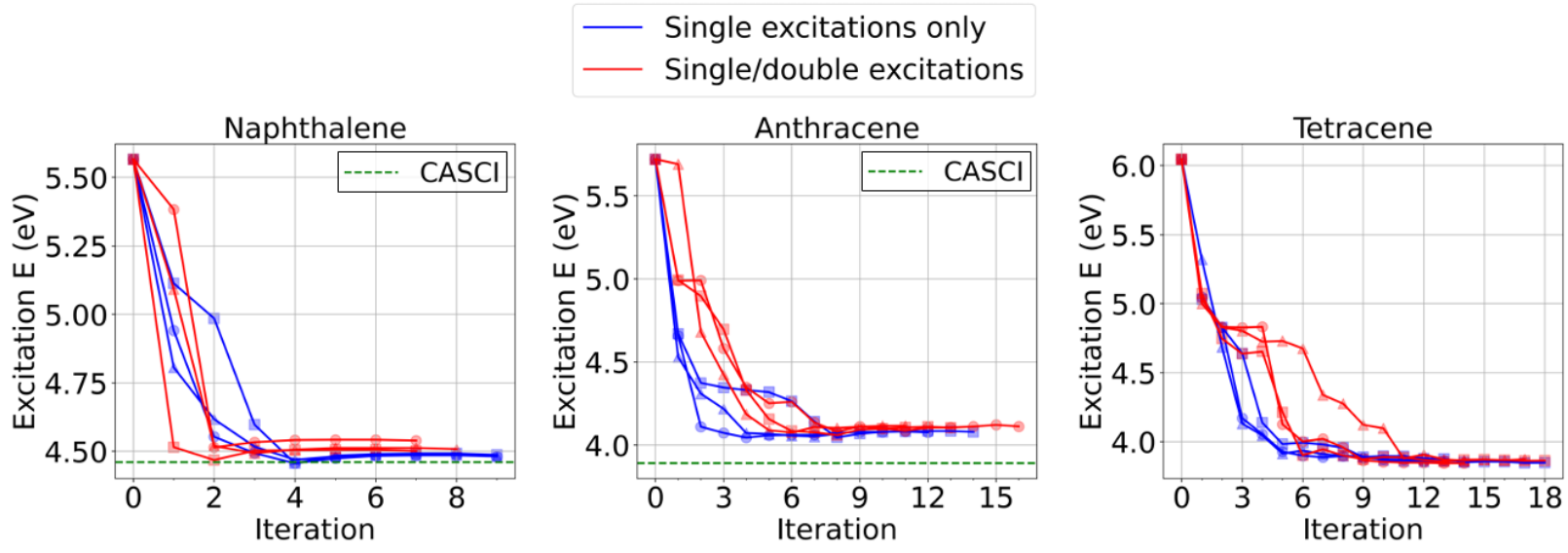

Figure 4. ALCI protocol convergences in terms of excitation energy depending on the maximum level of excitations for naphthalene, anthracene and tetracene: single excitations only vs. single/double excitations. Three independent protocol calculations (as indicated with different marker types) were performed for different maximum level of excitations. Iteration zero corresponds to the RASCI $(n=2)$ calculation.

We implemented a unique strategy to efficiently identify important configurations, which uses class probability (i.e., the probability of each class) of an ML classifier to decide which ML-predicted configurations should be labeled first. In general, an ML classifier predicts not only a class label (in our problem, important or unimportant) but also a probability for each class, namely a real number from 0 to 1 , with the sum of the probabilities being 1 . For a binary classification problem, a class is predicted to be positive or negative, respectively, if the class probability is larger or smaller, respectively, than a decision threshold, which we set to 0.5 , unless otherwise specified. For example, if a class probability for a given input (e.g., an unlabeled configuration) is predicted to be larger than 0.5 , then this class will be positive (e.g., important in our problem). Configurations with a high-class probability will most likely be important. 
Figure 5 shows the comparisons of protocol calculations based on using and not using class probabilities.

For naphthalene, both cases show similar convergence of the protocol calculations, resulting in the same excitation energy $(4.48 \mathrm{eV})$. However, as the system size increases, sampling queries based on the class probabilities outperform random query sampling, requiring a smaller number of iterations (from 14.3 to 11.3 iterations for anthracene, and from 28 to 15 iterations for tetracene on average) and producing slightly smaller excitation energies (from 4.10 to $4.07 \mathrm{eV}$ for anthracene, and from 3.90 to $3.86 \mathrm{eV}$ for tetracene on average).
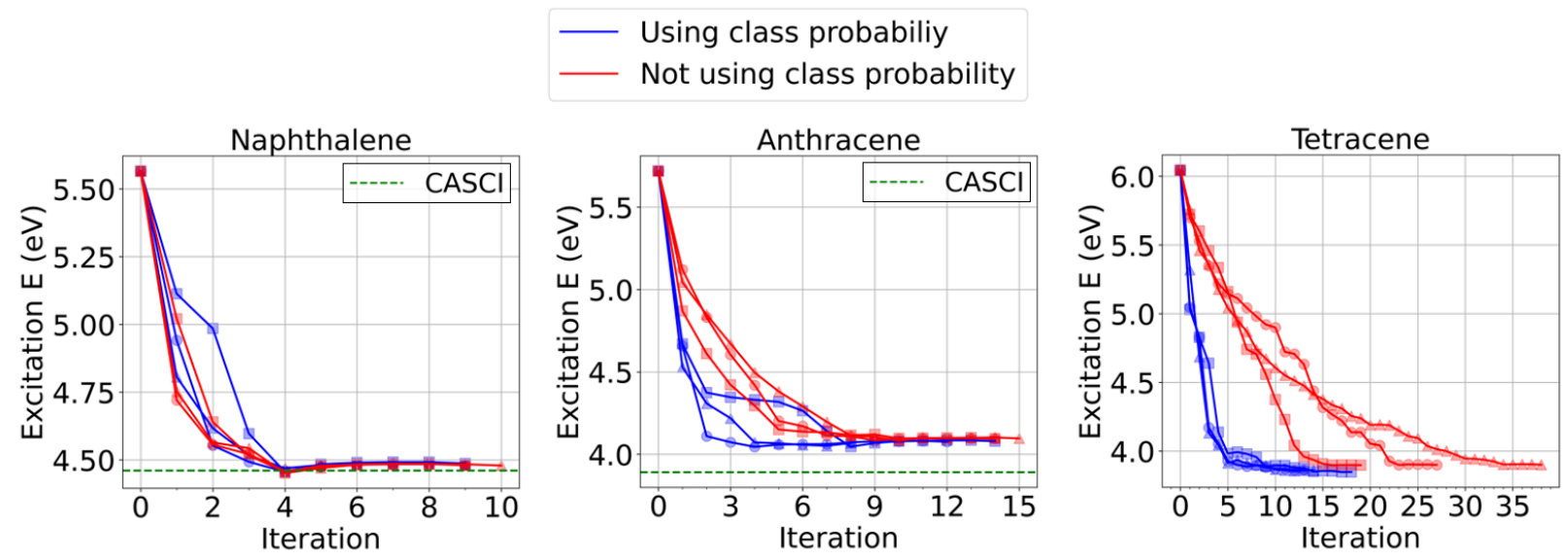

\section{- Based on ML prediction probabiliy Without ML prediction probability}
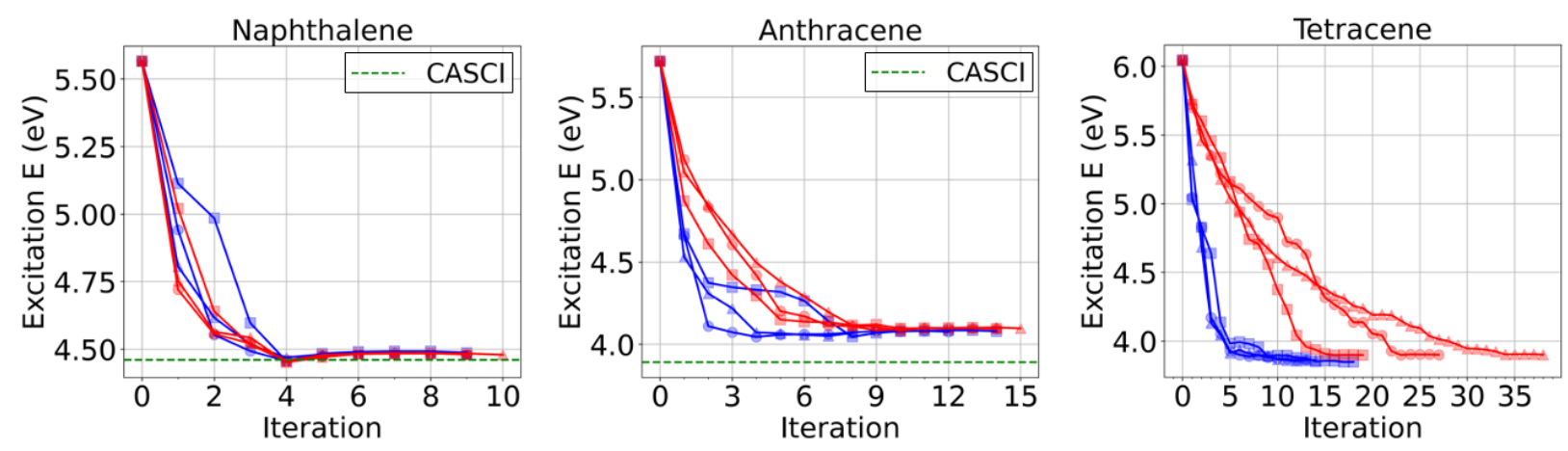

Figure 5. ALCI protocol convergences in terms of excitation energy depending on the use of class probability for query priority sampling for naphthalene, anthracene and tetracene. Three independent protocol calculations (as indicated with different marker types) were performed for each case. Iteration zero corresponds to the RASCI $(n=2)$ calculation. 


\subsection{Comparison of Different ML Algorithms}

Different ML algorithms including KRC, KNN, GP, RF, XGBoost and ANN were tested for the ALCI protocol. Interestingly, the convergence of the excitation energy in the ALCI protocol is highly dependent on both system size and type of the ML algorithm. For the smallest system, naphthalene, all the ML algorithms predicted a similar excitation energy $(4.48-4.50 \mathrm{eV})$. The $\mathrm{KRC}, \mathrm{KNN}$, GP and RF classifiers took about 8-9 ALCI iterations to converge, while XGBoost and ANN required about 11 and 13 iterations, respectively (Detailed ALCI results are available in the SI, section S6.1). This difference arises since there are not enough data to train the boosting algorithm and ANN many model-parameters and hyper-parameters, as demonstrated by low ML-model performance at early iterations for ANN and large fluctuations in the ML model performance for ANN and XGBoost (Variations of the ML model performance, F1, can be found in SI, Figure S8).

As the acene size increases, the performance of the different ML algorithms changes, affecting the ranking of the algorithms as far as the number of iterations is concerned (see SI, Figure S6 and Table S6). To clearly show this, the performance of the different ML algorithms for tetracene was evaluated, in terms of both number of iterations and computation time as listed in Table 1. For the evaluation of the computational time, five cores (3.00 GHz Intel i9-10980XE) were used if the ML model development tools (i.e., scikit-learn and XGBoost) support parallelization of the model training and prediction (i.e., for KNN, RF, and XGBoost), otherwise, one CPU (3.00 GHz Intel i9-10980XE) core is used (for GP and KRC). For ANN, a NVIDIA Quadro RTX 8000 graphics processing unit (GPU) was used. The ANN, GP, XGBoost and $\mathrm{KRC}$ algorithms require about 15 iterations, while the RF and $\mathrm{KNN}$ algorithms take 21 and 26 iterations, respectively. Although XGBoost and ANN require a similar number of iterations, as shown in Table 1, XGBoost is the fastest algorithm (about 3 hours on average for running an ALCI full cycle) while ANN is the slowest one (about 8 hours and a half on average) considering the overall computational time due to the time-consuming training procedure of ANN models even using the GPU. The KRC algorithm, which is 
using only one CPU core, exhibited a reasonably good performance, showing the second lowest computational cost (i.e., about 4 hours and a half). Although KRC showed different convergence trends in the excitation energy calculations, most of the calculations converged to very similar excitation energies (Figure S7). Compared to the KRC, GP employed ca. 20\% more time and converged to a slightly higher excitation energy. Like XGBoost, RF and KNN can also use multiple cores for parallel processing in ML model training, and these algorithms show faster convergence than XGBoost for naphthalene. However, for tetracene, RF and KNN took more iterations (i.e, 16 iterations for XGBoost vs. 21 and 26 iterations for $\mathrm{RF}$ and $\mathrm{KNN}$, respectively) and more computational time (about 3 hours for XGBoost vs. 4 hours 40 minutes and 6 hours 20 minutes for RF and KNN, respectively) than XGBoost. For the subsequent investigations, considering both the number of iterations and the computational time of the tested ML algorithms, we selected three ML algorithms, namely ANN (smallest numbers of iterations), XGBoost (fastest algorithm for tetracene), and KRC (it uses only one CPU core but shows good performance).

Table 1. ALCI protocol results for tetracene with different ML algorithms ${ }^{\mathrm{a}}$

\begin{tabular}{|c|c|c|c|c|}
\hline ML algorithm & $\begin{array}{c}\text { average number of } \\
\text { iterations }\end{array}$ & $\begin{array}{c}\text { number of important } \\
\text { CSFs }\end{array}$ & excitation energy $(\mathrm{eV})$ & wall time (hh:mm:ss) ${ }^{\mathrm{b}}$ \\
\hline ANN & 14.8 & 1625 & 3.88 & $08: 28: 34$ \\
\hline GP & 15.4 & 1642 & 3.96 & 05:30:10 \\
\hline XGBoost & 15.6 & 1753 & 3.87 & 03:01:12 \\
\hline $\mathrm{KRC}$ & 15.6 & 1749 & 3.90 & $04: 31: 11$ \\
\hline $\mathrm{RF}$ & 21.2 & 1781 & 3.88 & 04:41:09 \\
\hline $\mathrm{KNN}$ & 25.7 & 1724 & 3.91 & 06:19:01 \\
\hline
\end{tabular}

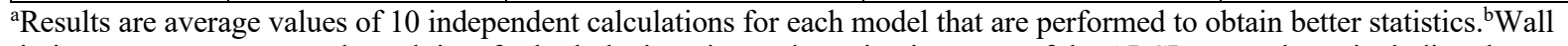
timings measure average elapsed time for both the iteration and termination steps of the ALCI protocol, not including the initialization step (i.e., DFT optimization, HF and RASCI $(n=2)$ calculations). To compare computational cost, the number of CPU cores for the calculations were limited to 5 cores (Intel i9-10980XE $3.00 \mathrm{GHz}$ ) if ML model training/predictions can be parallelized (i.e., for KNN, RF and XGBoost). For ANN, a GPU (NVIDIA Quadro RTX 8000) was used. GP and KRC models were trained and used with one CPU core (Intel i9-10980XE 3.00 GHz) due to the limitation of the software. 


\subsection{ALCI Results for Acenes and Pyrene}

\subsubsection{Active spaces up to $(16 \mathrm{e}, 160)$}

We applied the workflow presented in Figure 3 to compute the excitation energy of naphthalene. For this molecule, the $\pi$ and $\pi^{*}$ active space consists of 10 electrons in 10 orbitals. The CASCI $(10 \mathrm{e}, 10 \mathrm{o})$ result is compared with the ALCI results (Table 2). The CASCI (10e, 10o) corresponds to 19,404 CSFs $(63,504$ SDs) and predicts an excitation energy of $4.46 \mathrm{eV}$. In Figure 6 the ALCI convergence (using ANN, KRC, and XGBoost) using a CI coefficient threshold of 0.01 is shown. At iteration 0 (i.e., RASCI (n=2) calculation), the excitation energy is $5.57 \mathrm{eV}$, with about a hundred of important configurations. The excitation energy decreases in subsequent iterations, converging after 9-14 iterations. The converged excitation energies are between 4.48 to $4.50 \mathrm{eV}$. The number of important CSFs in the last iteration cycle is about 360 (about 4,000 SDs). It is impressive that the excitation energies are similar to the CASCI (10e, 10o) one, but with two orders of magnitude fewer CSFs. The excitation energy decreases upon iterating, which suggests that the newly included configurations improve the excited-state wave function more significantly than the ground-state wave function. This is not surprising, since the excited state is more multiconfigurational than the ground state, and therefore the former requires the inclusion of higher excitations for a more accurate description.

Anthracene has a $\pi-\pi^{*}$ active space active space of 14 electrons in 14 orbitals. The (14e, $\left.14 \mathrm{o}\right)$ CASCI $\left(2.76 \times 10^{6} \mathrm{CSF}\right.$ and $\left.1.12 \times 10^{7} \mathrm{SDs}\right)$ excitation energy is $3.89 \mathrm{eV}$. The excitation energies computed with ALCI are reported in Table 2 and Figure 6. The RASCI $(n=2)$ excitation energy (at iteration $=0)$ is 5.72 $\mathrm{eV}$, and then it decreases during the iterative procedure, reaching the value of $4.10 \mathrm{eV}$ after $10-12$ iterations. With the CI coefficient threshold of 0.01 , the ALCI excitation energy is about $0.2 \mathrm{eV}$ higher than the CASCI value, but it corresponds to only about $10^{3} \mathrm{CSFs}$ (about $2-4 \times 10^{4} \mathrm{SDs}$ ) in the last SCI calculation. So overall these calculations us three orders of magnitude fewer CSFs/SDs than the CASCI one. 
The pyrene molecule is a non-linear PAH and its active space includes 16 electrons in 16 orbitals. The CASCI $(16 \mathrm{e}, 16 \mathrm{o})$ corresponding to $3.48 \times 10^{7} \mathrm{CSFs}\left(1.66 \times 10^{8} \mathrm{SDs}\right)$ predicts and excitation energy of 3.79 eV. In Figure 6 the excitation energies obtained with ALCI are shown. The RASCI $(n=2)$ excitation energy is $5.98 \mathrm{eV}$, and the iterative protocol converges to about $4.0-4.15 \mathrm{eV}$ in $13-20$ iterations. By using the 0.01 threshold, the ALCI method provides an excitation energy within $0.3 \mathrm{eV}$ from CASCI, with the inclusion of only $1.2-1.4 \times 10^{3} \mathrm{CSFs},\left(4-4.5 \times 10^{4} \mathrm{SDs}\right)$, so four orders of magnitudes fewer than CASCI. Using a smaller threshold for the CI coefficients, 0.005, the predicted excitation energies are about 3.9 eV (i.e., a decrease of $0.1-0.16 \mathrm{eV}$ compared to using the threshold of 0.01 ), which are closer to the CASCI result. However, in return for accuracy, a larger number of iterations and important configurations is needed in the ALCI calculations, resulting in the increased computational cost (2.2-4.7 times depending on ML algorithms. See S6.3 for more details). It is observed that all the tested ML algorithms such as KRC, XGboost and ANN yield similar excitation energies.

Regarding timings, ALCI can be performed in about 22 minutes (using XGBoost with five Intel i910980XE@3.00 GHz)with respect to about 3 hours (using one Intel i9-10980XE@3.00 GHz) required for CASCI $(14 \mathrm{e}, 14 \mathrm{o})$ for anthracene. For pyrene, ALCI (using KRC and the CI coefficient threshold of 0.01) requires only about 46 minutes (using XGBoost with five Intel i9-10980XE@3.00 GHz), while CASCI requires more than 91 hours (using one Intel i9-10980XE@3.00 GHz). For larger active spaces, CASCI calculations become infeasible, while ALCI calculations are affordable. From the above ALCI results for naphthalene, anthracene, and pyrene, it is shown that the ALCI method is able to identify the important configurations needed to be included in the SCI calculations in few iteration cycles.

Table 2. ALCI protocol results with the optimized input parameters for naphthalene, anthracene, and pyrene $^{\mathrm{a}}$

\begin{tabular}{|c|c|c|c|c|c|c|}
\hline system & ML algorithm & $\begin{array}{c}\text { threshold for } \\
\text { CI coeff. }\end{array}$ & $\begin{array}{c}\text { average } \\
\text { number of } \\
\text { iterations }\end{array}$ & $\begin{array}{c}\text { number of important } \\
\text { CSFs }\end{array}$ & $\begin{array}{c}\text { number of important } \\
\text { SDs }\end{array}$ & $\begin{array}{c}\text { excitation } \\
\text { energy (eV) }\end{array}$ \\
\hline naphthalene & KRC & 0.01 & 9.0 & 369 & 4,104 & 4.48 \\
\hline
\end{tabular}




\begin{tabular}{|c|c|c|c|c|c|c|}
\hline system & ML algorithm & $\begin{array}{l}\text { threshold for } \\
\text { CI coeff. }\end{array}$ & $\begin{array}{c}\text { average } \\
\text { number of } \\
\text { iterations }\end{array}$ & $\begin{array}{c}\text { number of important } \\
\text { CSFs }\end{array}$ & $\begin{array}{c}\text { number of important } \\
\text { SDs }\end{array}$ & $\begin{array}{c}\text { excitation } \\
\text { energy }(\mathrm{eV})\end{array}$ \\
\hline \multirow[t]{6}{*}{$(10 \mathrm{e}, 10 \mathrm{o})$} & & 0.005 & 8.0 & 722 & 8,379 & 4.45 \\
\hline & \multirow{2}{*}{ ANN } & 0.01 & 13.3 & 356 & 3,828 & 4.50 \\
\hline & & 0.005 & 10.3 & 698 & 7,942 & 4.46 \\
\hline & \multirow{2}{*}{ XGBoost } & 0.01 & 10.7 & 362 & 4,072 & 4.48 \\
\hline & & 0.005 & 9.7 & 662 & 7,701 & 4.47 \\
\hline & \multicolumn{3}{|c|}{ CASCI $(10 \mathrm{e}, 10 \mathrm{o})$} & $19,404^{b}$ & $63,504^{b}$ & 4.46 \\
\hline \multirow{7}{*}{$\begin{array}{l}\text { anthracene } \\
(14 \mathrm{e}, 14 \mathrm{o})\end{array}$} & \multirow{2}{*}{$\mathrm{KRC}$} & 0.01 & 11.3 & 1,062 & 37,971 & 4.07 \\
\hline & & 0.005 & 11.7 & 2,474 & 100,328 & 3.97 \\
\hline & \multirow{2}{*}{ ANN } & 0.01 & 11.7 & 923 & 23,462 & 4.10 \\
\hline & & 0.005 & 10.3 & 2,353 & 78,577 & 3.98 \\
\hline & \multirow{2}{*}{ XGBoost } & 0.01 & 9.7 & 1,041 & 37,278 & 4.07 \\
\hline & & 0.005 & 12.3 & 2,328 & 97,536 & 4.01 \\
\hline & \multicolumn{3}{|c|}{ CASCI $(14 \mathrm{e}, 14 \mathrm{o})$} & $2,760,615^{\mathrm{b}}$ & $11,778,624^{\mathrm{b}}$ & 3.89 \\
\hline \multirow{7}{*}{$\begin{array}{c}\text { pyrene } \\
(16 \mathrm{e}, 160)\end{array}$} & \multirow{2}{*}{$\mathrm{KRC}$} & 0.01 & 13.7 & 1,444 & 41,961 & 4.13 \\
\hline & & 0.005 & 16.7 & 3,660 & 225,039 & 3.98 \\
\hline & \multirow{2}{*}{ ANN } & 0.01 & 14.7 & 1,243 & 45,457 & 4.15 \\
\hline & & 0.005 & 15.7 & 3,424 & 151,508 & 3.99 \\
\hline & \multirow{2}{*}{ XGBoost } & 0.01 & 12.7 & 1,407 & 40,522 & 4.14 \\
\hline & & 0.005 & 20.0 & 3,505 & 155,884 & 4.00 \\
\hline & \multicolumn{3}{|c|}{ CASCI (16e, 16o) } & $34,763,300^{\mathrm{b}}$ & $165,636,900^{\mathrm{b}}$ & 3.79 \\
\hline
\end{tabular}

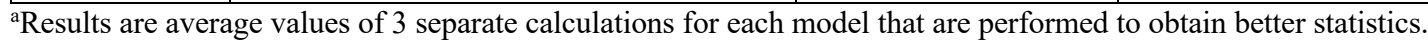

${ }^{b}$ Total number of configurations or determinants in the active space.

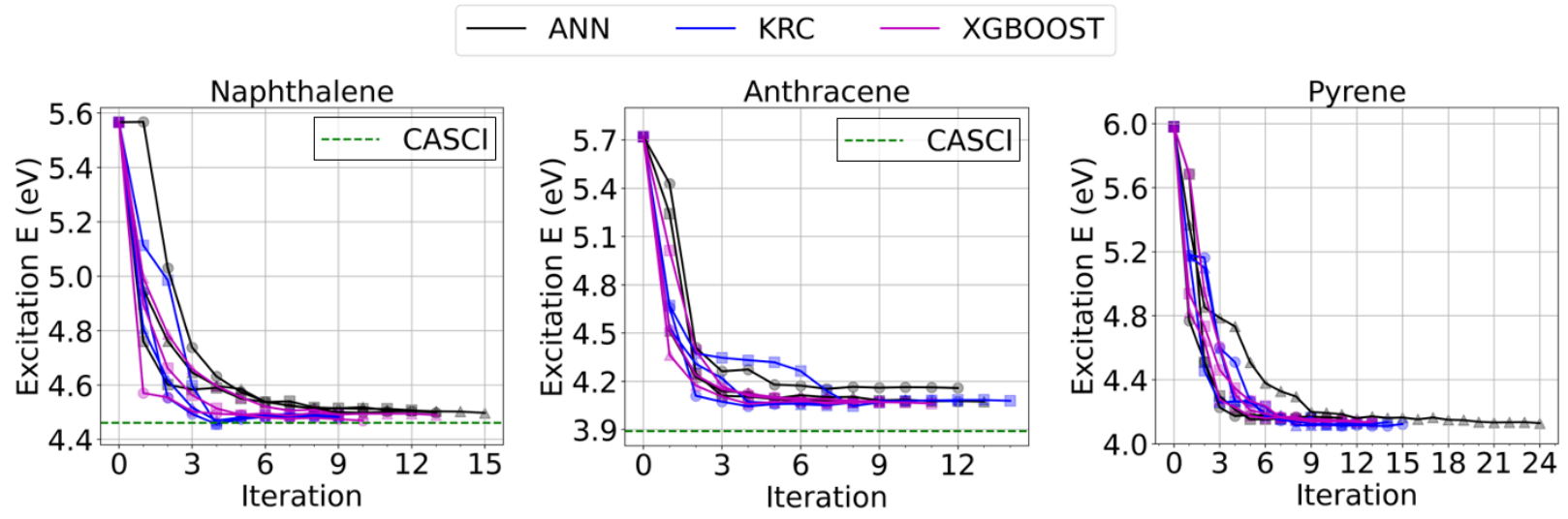

Figure 6. The ALCI protocol convergence in terms of excitation energy for naphthalene, anthracene, and pyrene. Three independent calculations (as indicated with different marker types) are performed for each model. CI coefficient threshold for important configuration is 0.01 . Iteration zero corresponds to the RASCI $(n=2)$ calculation. 
The ground and excited state wave functions for anthracene and pyrene have then been analyzed by comparing the active orbitals occupation numbers obtained with ALCI and CASCI. These values are reported in Section S6. The differences in occupation numbers between ALCI and CASCI are within 0.03, pointing to accurate ALCI wave functions.

\subsubsection{Active spaces beyond $(16 e, 160)$}

We used the ALCI method to compute excitation energies for larger active spaces, for which the respective CASCI calculation are not affordable, and the results are reported in Table 3 and Figure 7. Tetracene, pentacene and hexacene have been investigated, with active spaces of (18e, 18o), (22e, 22o) and (26e, 26o), respectively. While CASCI calculations are not feasible, experimental data are available, and therefore they have been used as benchmarks for the ALCI results.

For tetracene, ALCI with a CI coefficient threshold of 0.01 yields an excitation energy of about $3.9 \mathrm{eV}$ in $10-15$ cycles, including about $1.5-1.7 \times 10^{3} \mathrm{CSFs}\left(3-5 \times 10^{4} \mathrm{SDs}\right)$. A decrease of about $0.1-0.2 \mathrm{eV}$ in the predicted excitation energy is achievable by decreasing the CI coefficient threshold to 0.005 at the expense of computation cost (i.e., 6-9.4 times). For pentacene, ALCI converges to an excitation energy of about $3.4-3.5 \mathrm{eV}$ in $11-17$ iterations, including about $1.7-2 \times 10^{3}$ configurations $\left(2-3 \times 10^{4}\right.$ determinants $)$ in the wave function when the 0.01 threshold is chosen. Lastly, in the case of hexacene, ALCI converges to an excitation energy of about $2.9 \mathrm{eV}$ in $12-24$ iterations, including only about $2.4-2.7 \times 10^{3} \mathrm{CSFs}\left(2.5-5.1 \times 10^{4}\right.$ SDs) in the wave function. Considering that the number of determinants for a CASCI (26e, 26o) calculation is about $1.08 \times 10^{14}$, ALCI can reduce this number by ten orders of magnitude, making this calculation feasible with a reasonable computational time (about 19 hours using KRC and ANN, 50 hours with XGBoost. See SI, section S6.6). Unexpectedly, we see that excitation energies using ALCI with the lower threshold of 0.005 showed almost no improvement for pentacene, and even deterioration for hexacene. This could happen if less relevant configurations (i.e, configurations that only marginally contribute to the overall energy) from the vast CASCI configuration space are included in the training set for each iteration 
by lowering the CI coefficient threshold for such a large system. To overcome this issue for larger systems, different featurization methods and advanced ranking strategies for choosing queries need to be developed and integrated with our ALCI protocol in future work.

Table 3. ALCI protocol results with the optimized input parameters for tetracene, pentacene, and hexacene ${ }^{\text {a. }}$

\begin{tabular}{|c|c|c|c|c|c|c|}
\hline system & $\begin{array}{c}\text { ML } \\
\text { algorithm }\end{array}$ & $\begin{array}{l}\text { threshold for } \\
\text { CI coeff. }\end{array}$ & $\begin{array}{l}\text { average } \\
\text { number of } \\
\text { iterations }\end{array}$ & $\begin{array}{c}\text { number of important } \\
\text { CSFs }\end{array}$ & $\begin{array}{c}\text { number of important } \\
\text { SDs }\end{array}$ & $\begin{array}{c}\text { excitation } \\
\text { energy }(\mathrm{eV})\end{array}$ \\
\hline \multirow{7}{*}{$\begin{array}{l}\text { tetracene } \\
(18 \mathrm{e}, 18 \mathrm{o})\end{array}$} & \multirow{2}{*}{$\mathrm{KRC}$} & 0.01 & 15.0 & 1,759 & 53,213 & 3.86 \\
\hline & & 0.005 & 22.7 & 4,788 & 251,320 & 3.74 \\
\hline & \multirow{2}{*}{ ANN } & 0.01 & 10.3 & 1,491 & 32,663 & 3.91 \\
\hline & & 0.005 & 18.0 & 3,944 & 179,952 & 3.73 \\
\hline & \multirow{2}{*}{ XGBoost } & 0.01 & 15.0 & 1,741 & 53,125 & 3.88 \\
\hline & & 0.005 & 24.3 & 4,642 & 234,622 & 3.75 \\
\hline & \multicolumn{3}{|c|}{ CASCI $(18 \mathrm{e}, 18 \mathrm{o})$} & $449,141,836^{\mathrm{b}}$ & $2,363,904,400^{\mathrm{b}}$ & $\mathrm{N} / \mathrm{A}$ \\
\hline \multirow{7}{*}{$\begin{array}{l}\text { pentacene } \\
(22 \mathrm{e}, 22 \mathrm{o})\end{array}$} & \multirow{2}{*}{$\mathrm{KRC}$} & 0.01 & 12.7 & 1,793 & 31,491 & 3.50 \\
\hline & & 0.005 & 25.0 & 4,780 & 216,678 & 3.46 \\
\hline & \multirow{2}{*}{ ANN } & 0.01 & 10.7 & 1,713 & 22,622 & 3.44 \\
\hline & & 0.005 & 16.0 & 4,195 & 175,336 & 3.48 \\
\hline & \multirow{2}{*}{ XGBoost } & 0.01 & 17.0 & 1,979 & 47,645 & 3.47 \\
\hline & & 0.005 & 25.0 & 4,941 & 231,838 & 3.46 \\
\hline & \multicolumn{3}{|c|}{ CASCI $(22 \mathrm{e}, 22 \mathrm{o})$} & $79,483,257,308^{b}$ & $497,634,306,624^{\mathrm{b}}$ & $\mathrm{N} / \mathrm{A}$ \\
\hline \multirow{7}{*}{$\begin{array}{l}\text { hexacene } \\
(26 \mathrm{e}, 26 \mathrm{o})\end{array}$} & \multirow{2}{*}{$\mathrm{KRC}$} & 0.01 & 17.3 & 2,430 & 27,468 & 2.89 \\
\hline & & 0.005 & 18.7 & 4,061 & 58,237 & 3.02 \\
\hline & \multirow{2}{*}{ ANN } & 0.01 & 12.3 & 2,366 & 24,760 & 2.89 \\
\hline & & 0.005 & 15.0 & 4,574 & 101,015 & 3.03 \\
\hline & \multirow{2}{*}{ XGBoost } & 0.01 & 24.0 & 2,670 & 51,247 & 2.93 \\
\hline & & 0.005 & 36.3 & 5,952 & 245,534 & 3.03 \\
\hline & \multicolumn{3}{|c|}{ CASCI (26e, 26o) } & $14,901,311,070,000^{b}$ & $108,172,480,360,000^{b}$ & N/A \\
\hline
\end{tabular}

${ }^{a}$ Results are average values of 3 separate calculations for each model that are performed to obtain better statistics.

${ }^{\mathrm{b}}$ Total number of configurations or determinants in the active space. 

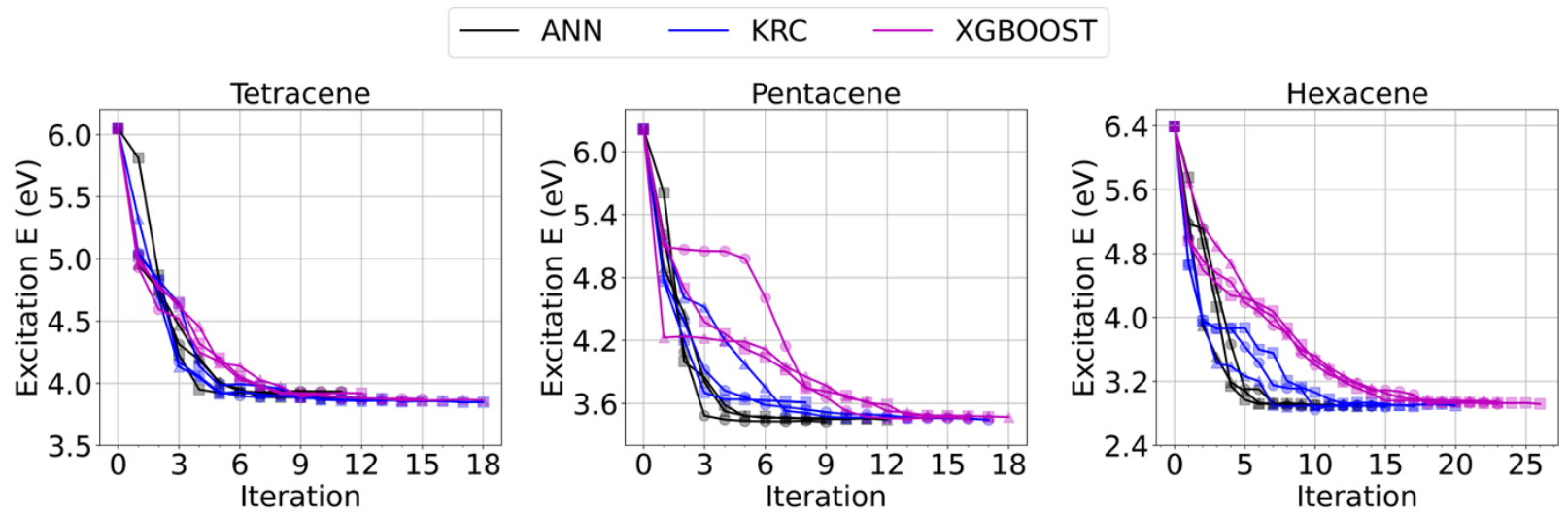

Figure 7. The ALCI protocol convergence in terms of excitation energy for tetracene, pentacene, and hexacene. Three separate calculations (as indicated with different marker types) are performed for each model. CI coefficient threshold for important configuration is 0.01 . The iteration zero corresponds to the RASCI ( $\mathrm{n}=2)$ calculation.

With increasing acene size, the computational time for the ML model training/predictions becomes negligibly small while the computational cost for the SCI calculations grows especially when using KRC or XGBoost (detailed timings data for arbitrary selected ALCI calculations are reported SI, section S6.7). For example, the relative computational costs for the ML model training/predictions and SCI calculations for hexacene are $3.2 \%$ and $96.6 \%$, respectively, when using $\mathrm{KRC}$, and $0.8 \%$ and $99.0 \%$, respectively, when using XGBoost. In the case of ANN, although the time of the SCI calculations increases with the acene size, for hexacene about half of the time is spent in the ANN model training (i.e., 48.7\%). Regarding the overall computational cost for the ALCI protocol, XGBoost, which is the fastest algorithm for tetracene, becomes the slowest one among the three ML algorithms tested for hexacene (about 50 hours on average using five Intel i9-10980XE @3.00 GHz). Figure 7 shows that XGBoost cannot identify important configurations effectively as the iterations proceed compared to the KRC and ANN algorithms, resulting in larger (average) numbers of important configurations (i.e., 2670 for XGBoost vs. ca. 2400 for KRC and ANN) and iterations (about 24, 17, and 12 iterations for XGBoost, KRC and ANN, respectively) than other ML algorithms. On the other hand, ANN performs better as the acene size increases, leading to a similar computational cost of about19 hours compared to the fastest ML algorithm, KRC (See Supporting 
Information, Table S13). This trend indicates that ANN would be the best performing ML algorithm in the ALCI method for systems with active spaces larger than (26e, 26o).

\subsubsection{Comparison with experimental data}

Finally, the excitation energies using ALCI (with KRC, XGBoost and ANN as ML algorithms) and CASCI, are compared to experimental values in Figure $8{ }^{87}$ For all the acenes analyzed in this work, there are two possible lowest singlet excited states depending on the orbitals that take part in the excitation process. One of them is labeled $L_{\mathrm{a}}$, corresponding to a HOMO to LUMO excitation, while the other $\left(L_{\mathrm{b}}\right)$ arises from a mixture of the HOMO-1 to LUMO and the HOMO to LUMO+1 excitations. ${ }^{66,87,88}$ The computed lowest excited states with different methods (along with the experimental ones) are shown in Table 4. Note that ALCI method yields almost the same excitation energy using the three different ML algorithms. The ALCI and CASCI lowest excited state is $L_{\mathrm{b}}$ for both naphthalene and anthracene. The CASCI method recovers only part of electron correlation, and therefore a subsequent calculation on top of the CASCI wave function is required to compute accurate energies. This is usually done using perturbation theory (PT2) $)^{89,90}$ or the more recent pair-density functional theory (PDFT) ${ }^{91}$ starting from a multiconfigurational wave function. We thus performed a PT2 calculation on top of CASCI, CASCI+PT2, to compute excitation energies using the computational procedure reported in Section S8. The lowest excited state for anthracene is now $L_{\mathrm{a}}$, as experimentally found. Moreover, we notice that the PT2 correction does not change sizably the $L_{\mathrm{b}}$ excitation energy, but it lowers the $L_{\mathrm{a}}$ energy by about $1 \mathrm{eV}$, with respect to CASCI. ALCI and CASCI predict the same excitation energy for naphthalene, while ALCI yields a higher excitation energy than CASCI (by about $0.2 \mathrm{eV}$ ) for anthracene. For larger acenes, CASCI calculations are not affordable, and therefore we compare ALCI results only with experiments. For the series of acenes analyzed, the computed excitation energy decreases with the acene length, reproducing the experimental trend. The ALCI excitation energies overestimate the experimental ones by about $0.3 \mathrm{eV}$ when $L_{\mathrm{b}}$ is the lowest excited state. The ALCI and experimental values discrepancy is higher (about $0.8 \mathrm{eV}$ ) when $L_{\mathrm{a}}$ is the lowest excited state. We notice that the ALCI excitation energies of $L_{\mathrm{a}}$ states are higher with respect to the experimental ones, but the PT2 
correction would lower them, based on the naphthalene and anthracene CASCI+PT2 results, and therefore this correction will be needed to achieve higher accuracy. The important finding is that the ALCI results can reproduce the experimental trend and, since the ALCI wave functions are similar to the CASCI ones, but they are considerably less expensive, (Section S7), they can be used as starting points for subsequent PT2 or PDFT calculations.

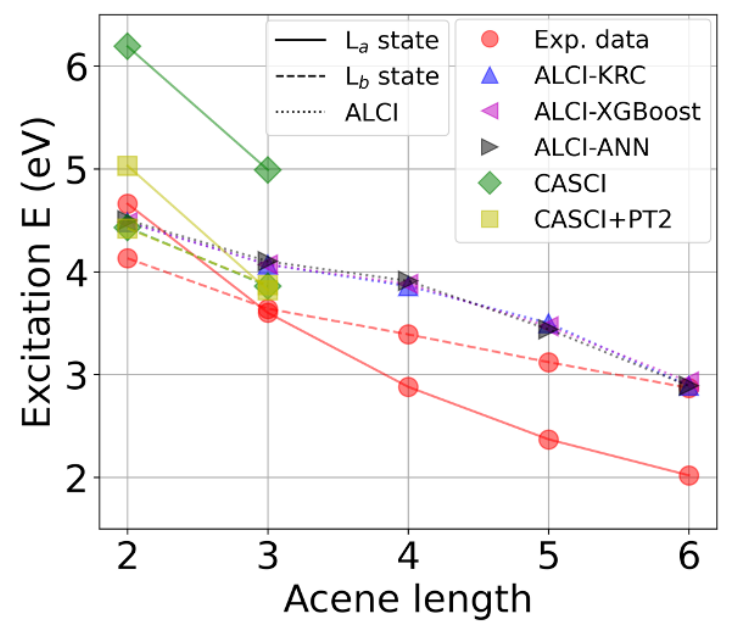

Figure 8. Excitation energy for different acene lengths computed with ALCI (using KRC, XGBoost and ANN as ML algorithms) and CASCI. Experimental results from Ref. 84 are also reported.

Table 4. First singlet vertical excited states for acenes determined by different methods

\begin{tabular}{|c|c|c|c|c|c|}
\hline & naphthalene & anthracene & tetracene & pentacene & hexacene \\
\hline exp. data & $\mathrm{L}_{\mathrm{b}}$ & $\mathrm{L}_{\mathrm{a}}$ & $\mathrm{L}_{\mathrm{a}}$ & $\mathrm{L}_{\mathrm{a}}$ & $\mathrm{L}_{\mathrm{a}}$ \\
\hline CASCI & $\mathrm{L}_{\mathrm{b}}$ & $\mathrm{L}_{\mathrm{b}}$ & $\mathrm{N} / \mathrm{A}$ & $\mathrm{N} / \mathrm{A}$ & N/A \\
\hline CASCI + PT2 & $\mathrm{L}_{\mathrm{b}}$ & $\mathrm{L}_{\mathrm{a}}$ & $\mathrm{N} / \mathrm{A}$ & $\mathrm{N} / \mathrm{A}$ & N/A \\
\hline ALCI & $\mathrm{L}_{\mathrm{b}}$ & $\mathrm{L}_{\mathrm{b}}$ & $\mathrm{L}_{\mathrm{b}}$ & $\mathrm{L}_{\mathrm{a}}$ & $\mathrm{L}_{\mathrm{a}}$ \\
\hline
\end{tabular}

\section{Conclusions}


We developed an iterative active machine learning method, called the active learning configuration interaction (ALCI) method, that can be used to efficiently identify important configurations in large active spaces calculations. We tested the ALCI method to compute the first singlet excited state of naphthalene, anthracene, tetracene, pentacene, hexacene, and pyrene. ALCI can identify the most important configurations within 10-20 iterations, yielding excitation energies that differ at most by $0.3 \mathrm{eV}$ from the CASCI ones, but with orders of magnitude fewer configurations. We employed ALCI to calculate excitation energies for active spaces up to $(26 \mathrm{e}, 260)$, for which CASCI is unfeasible. In the cases where we could not perform the CASCI calculation, we compared ALCI excitation energies to the experimental ones and found that ALCI is able to reproduce the experimental trend, namely the lowering of the excitation energy with

the increasing acene length. For hexacene, ALCI converges with only about 2,400 configuration state functions $(25,000$ Slater determinants $)$ in the wave function, with a reduction of the number of determinants of about 10 orders of magnitude with respect to the corresponding CASCI (26e, 26o). This study shows that, among the various ML algorithms tested, namely KRC, KNN, GP, RF, XGBoost, and ANN, the ANN model exhibits the best ALCI performance in terms of both fewer iterations and lowest computational cost of about $19 \mathrm{hr}$ for the largest system, hexacene. Integrating ALCI protocol with recently developed efficient CI solving algorithms ${ }^{39,92-94}$ could enable us to investigate even larger active spaces. Lastly the ALCI wave functions can be used as starting point for PT2 or PDFT subsequent calculations to achieve higher accuracy in predicting excitation energies.

\section{Acknowledgements}

This work was supported as part of the Nanoporous Materials Genome Center, funded by the U.S. Department of Energy, Office of Basic Energy Sciences, Division of Chemical Sciences, Geosciences and Biosciences under Award DE-FG02-17ER16362. Computer resources were provided by the Minnesota Supercomputing Institute at the University of Minnesota. 


\section{ORCID}

WooSeok Jeong: 0000-0003-3885-8494

Carlo Alberto Gaggioli: 0000-0001-9105-8731

Laura Gagliardi: 0000-0001-5227-1396

\section{Corresponding authors}

lgagliardi@uchicago.edu

\section{Competing interests}

The authors declare no competing interests.

\section{References}

(1) Hedley, G. J.; Ruseckas, A.; Samuel, I. D. W. Light Harvesting for Organic Photovoltaics. Chem. Rev. 2017, 117, 796-837.

(2) Liu, W.-H.; Wu, I.-C.; Lai, C.-H.; Lai, C.-H.; Chou, P.-T.; Li, Y.-T.; Chen, C.-L.; Hsu, Y.-Y.; Chi, Y. Simple Organic Molecules Bearing a 3, 4-Ethylenedioxythiophene Linker for Efficient DyeSensitized Solar Cells. Chem. Commun. 2008, No. 41, 5152-5154.

(3) Zhang, L.; Yang, X.; Wang, W.; Gurzadyan, G. G.; Li, J.; Li, X.; An, J.; Yu, Z.; Wang, H.; Cai, B.; Hagfeldt, A.; Sun, L. 13.6\% Efficient Organic Dye-Sensitized Solar Cells by Minimizing Energy Losses of the Excited State. ACS Energy Lett. 2019, 4, 943-951.

(4) Zhou, Y.; He, Q.; Yang, Y.; Zhong, H.; He, C.; Sang, G.; Liu, W.; Yang, C.; Bai, F.; Li, Y. Binaphthyl-containing Green-and Red-emitting Molecules for Solution-processable Organic Light-emitting Diodes. Adv. Funct. Mater. 2008, 18, 3299-3306. 
(5) Kalinowski, J. Organic Light-Emitting Diodes: Principles, Characteristics \& Processes; CRC press, 2018.

(6) Oh, Y.; Hu, X. Organic Molecules as Mediators and Catalysts for Photocatalytic and Electrocatalytic $\mathrm{CO}_{2}$ Reduction. Chem. Soc. Rev. 2013, 42, 2253-2261.

(7) Theriot, J. C.; Lim, C.-H.; Yang, H.; Ryan, M. D.; Musgrave, C. B.; Miyake, G. M. Organocatalyzed Atom Transfer Radical Polymerization Driven by Visible Light. Science. 2016, $352,1082-1086$.

(8) Griesbeck, A.; Oelgemöller, M.; Ghetti, F. CRC Handbook of Organic Photochemistry and Photobiology, -Two Volume Set; CRC press, 2019.

(9) Itoh, T. Fluorescence and Phosphorescence from Higher Excited States of Organic Molecules. Chem. Rev. 2012, 112, 4541-4568.

(10) Chen, D.; Liu, C.; Tang, J.; Luo, L.; Yu, G. Fluorescent Porous Organic Polymers. Polym. Chem. 2019, 10, 1168-1181.

(11) Haug, W. K.; Moscarello, E. M.; Wolfson, E. R.; McGrier, P. L. The Luminescent and Photophysical Properties of Covalent Organic Frameworks. Chem. Soc. Rev. 2020, 49, 839-864.

(12) Adamo, C.; Jacquemin, D. The Calculations of Excited-State Properties with Time-Dependent Density Functional Theory. Chem. Soc. Rev. 2013, 42, 845-856.

(13) Jacquemin, D.; Wathelet, V.; Perpete, E. A.; Adamo, C. Extensive TD-DFT Benchmark: SingletExcited States of Organic Molecules. J. Chem. Theory Comput. 2009, 5, 2420-2435.

(14) Laurent, A. D.; Jacquemin, D. TD-DFT Benchmarks: A Review. Int. J. Quantum Chem. 2013, $113,2019-2039$.

(15) Elliott, P.; Furche, F.; Burke, K. 3 Excited States from Time-Dependent Density Functional 
Theory. Rev. Comput. Chem. 2009, 26, 91.

(16) Mardirossian, N.; Head-Gordon, M. Thirty Years of Density Functional Theory in Computational Chemistry: An Overview and Extensive Assessment of 200 Density Functionals. Mol. Phys. 2017, $115,2315-2372$.

(17) Kedziora, G. S.; Barr, S. A.; Berry, R.; Moller, J. C.; Breitzman, T. D. Bond Breaking in Stretched Molecules: Multi-Reference Methods versus Density Functional Theory. Theor. Chem. Acc. 2016, $135,79$.

(18) Sherrill, C. D.; Dutta, A.; Abrams, M. L.; Sears, J. S. Bond Breaking in Quantum Chemistry: A Comparison of Single-and Multi-Reference Methods; ACS Publications, 2007.

(19) Venturinelli Jannuzzi, S. A.; Phung, Q. M.; Domingo, A.; Formiga, A. L. B.; Pierloot, K. Spin State Energetics and Oxyl Character of Mn-Oxo Porphyrins by Multiconfigurational Ab Initio Calculations: Implications on Reactivity. Inorg. Chem. 2016, 55, 5168-5179.

(20) Gaggioli, C. A.; Stoneburner, S. J.; Cramer, C. J.; Gagliardi, L. Beyond Density Functional Theory: The Multiconfigurational Approach to Model Heterogeneous Catalysis. ACS Catal. 2019, 9, 8481-8502.

(21) Roemelt, M.; Pantazis, D. A. Multireference Approaches to Spin-State Energetics of Transition Metal Complexes Utilizing the Density Matrix Renormalization Group. Adv. Theory Simulations 2019, 2, 1800201.

(22) Roos, B. O. Multiconfigurational Quantum Chemistry for Ground and Excited States. In Radiation induced molecular phenomena in nucleic acids; Springer, 2008; pp 125-156.

(23) Lischka, H.; Nachtigallová, D.; Aquino, A. J. A.; Szalay, P. G.; Plasser, F.; Machado, F. B. C.; Barbatti, M. Multireference Approaches for Excited States of Molecules. Chem. Rev. 2018, 118, 7293-7361. 
(24) Mok, D. K. W.; Neumann, R.; Handy, N. C. Dynamical and Nondynamical Correlation. J. Phys. Chem. 1996, 100, 6225-6230.

(25) Hollett, J. W.; Gill, P. M. W. The Two Faces of Static Correlation. J. Chem. Phys. 2011, 134, 114111.

(26) Roos, B. O.; Taylor, P. R.; Sigbahn, P. E. M. A Complete Active Space SCF Method (CASSCF) Using a Density Matrix Formulated Super-CI Approach. Chem. Phys. 1980, 48, 157-173.

Aquilante, F.; Autschbach, J.; Carlson, R. K.; Chibotaru, L. F.; Delcey, M. G.; De Vico, L.; Fdez. Galván, I.; Ferré, N.; Frutos, L. M.; Gagliardi, L.; Garavelli, M.; Giussani, A.; Hoyer, C. E.; Li Manni, G.; Lischka, H.; Ma, D.; Malmqvist, P. Å.; Müller, T.; Nenov, A.; Olivucci, M.; Pedersen, T. B.; Peng, D.; Plasser, F.; Pritchard, B.; Reiher, M.; Rivalta, I.; Schapiro, I.; Segarra-Martí, J.; Stenrup, M.; Truhlar, D. G.; Ungur, L.; Valentini, A.; Vancoillie, S.; Veryazov, V.; Vysotskiy, V. P.; Weingart, O.; Zapata, F.; Lindh, R. Molcas 8: New Capabilities for Multiconfigurational Quantum Chemical Calculations across the Periodic Table. J. Comput. Chem. 2016, 37, 506-541.

(28) Vogiatzis, K. D.; Ma, D.; Olsen, J.; Gagliardi, L.; De Jong, W. A. Pushing ConfigurationInteraction to the Limit: Towards Massively Parallel MCSCF Calculations. J. Chem. Phys. 2017, $147,184111$.

(29) Malmqvist, P. Å.; Pierloot, K.; Shahi, A. R. M.; Cramer, C. J.; Gagliardi, L. The Restricted Active Space Followed by Second-Order Perturbation Theory Method: Theory and Application to the Study of $\mathrm{Cu} \mathrm{O}_{2}$ and $\mathrm{Cu}_{2} \mathrm{O}_{2}$ Systems. J. Chem. Phys. 2008, 128, 204109.

(30) Ma, D.; Li Manni, G.; Gagliardi, L. The Generalized Active Space Concept in Multiconfigurational Self-Consistent Field Methods. J. Chem. Phys. 2011, 135, 44128.

(31) Hermes, M. R.; Gagliardi, L. Multiconfigurational Self-Consistent Field Theory with Density Matrix Embedding: The Localized Active Space Self-Consistent Field Method. J. Chem. Theory 
Comput. 2019, 15, 972-986.

(32) Schriber, J. B.; Evangelista, F. A. Communication: An Adaptive Configuration Interaction Approach for Strongly Correlated Electrons with Tunable Accuracy. J. Chem. Phys. 2016, 144, 161106.

(33) Liu, W.; Hoffmann, M. R. ICI: Iterative CI toward Full CI. J. Chem. Theory Comput. 2016, 12, $1169-1178$.

(34) Zimmerman, P. M. Incremental Full Configuration Interaction. J. Chem. Phys. 2017, 146, 104102.

Garniron, Y.; Scemama, A.; Loos, P.-F.; Caffarel, M. Hybrid Stochastic-Deterministic Calculation of the Second-Order Perturbative Contribution of Multireference Perturbation Theory. J. Chem. Phys. 2017, 147, 34101.

(36) Mejuto-Zaera, C.; Tubman, N. M.; Whaley, K. B. Dynamical Mean Field Theory Simulations with the Adaptive Sampling Configuration Interaction Method. Phys. Rev. B 2019, 100, 125165.

(37) Huron, B.; Malrieu, J. P.; Rancurel, P. Iterative Perturbation Calculations of Ground and Excited State Energies from Multiconfigurational Zeroth-order Wavefunctions. J. Chem. Phys. 1973, 58, $5745-5759$.

(38) Tubman, N. M.; Lee, J.; Takeshita, T. Y.; Head-Gordon, M.; Whaley, K. B. A Deterministic Alternative to the Full Configuration Interaction Quantum Monte Carlo Method. J. Chem. Phys. 2016, 145,44112 .

(39) Levine, D. S.; Hait, D.; Tubman, N. M.; Lehtola, S.; Whaley, K. B.; Head-Gordon, M. CASSCF with Extremely Large Active Spaces Using the Adaptive Sampling Configuration Interaction Method. J. Chem. Theory Comput. 2020, 16, 2340-2354.

(40) Holmes, A. A.; Tubman, N. M.; Umrigar, C. J. Heat-Bath Configuration Interaction: An Efficient Selected Configuration Interaction Algorithm Inspired by Heat-Bath Sampling. J. Chem. Theory 
Comput. 2016, 12, 3674-3680.

(41) Greer, J. C. Estimating Full Configuration Interaction Limits from a Monte Carlo Selection of the Expansion Space. J. Chem. Phys. 1995, 103, 1821-1828.

(42) Greer, J. C. Monte Carlo Configuration Interaction. J. Comput. Phys. 1998, 146, 181-202.

(43) Tong, L.; Nolan, M.; Cheng, T.; Greer, J. C. Monte Carlo Configuration Generation Computer Program for the Calculation of Electronic States of Atoms, Molecules, and Quantum Dots.

Comput. Phys. Commun. 2000, 131, 142-163.

(44) Dral, P. O. Quantum Chemistry in the Age of Machine Learning. J. Phys. Chem. Lett. 2020, 11, $2336-2347$.

(45) Rupp, M. Machine Learning for Quantum Mechanics in a Nutshell. Int. J. Quantum Chem. 2015, $115,1058-1073$.

(46) Noé, F.; Tkatchenko, A.; Müller, K.-R.; Clementi, C. Machine Learning for Molecular Simulation. Annu. Rev. Phys. Chem. 2020, 71, 361-390.

(47) Von Lilienfeld, O. A.; Müller, K.-R.; Tkatchenko, A. Exploring Chemical Compound Space with Quantum-Based Machine Learning. Nat. Rev. Chem. 2020, 4, 347-358.

(48) Ramakrishnan, R.; von Lilienfeld, O. A. Machine Learning, Quantum Chemistry, and Chemical Space. Rev. Comput. Chem. 2017, 30, 225-256.

(49) Von Lilienfeld, O. A. Quantum Machine Learning in Chemical Compound Space. Angew. Chemie Int. Ed. 2018, 57, 4164-4169.

(50) Townsend, J.; Vogiatzis, K. D. Data-Driven Acceleration of the Coupled-Cluster Singles and Doubles Iterative Solver. J. Phys. Chem. Lett. 2019, 10, 4129-4135.

(51) Townsend, J.; Vogiatzis, K. D. Transferable MP2-Based Machine Learning for Accurate Coupled- 
Cluster Energies. J. Chem. Theory Comput. 2020, 16, 7453-7461.

(52) Westermayr, J.; Marquetand, P. Machine Learning for Electronically Excited States of Molecules. Chem. Rev. 2020. https://doi.org/10.1021/acs.chemrev.0c00749.

(53) Schütt, K. T.; Gastegger, M.; Tkatchenko, A.; Müller, K.-R.; Maurer, R. J. Unifying Machine Learning and Quantum Chemistry with a Deep Neural Network for Molecular Wavefunctions. Nat. Commun. 2019, 10, 1-10.

(54) Settles, B. Active Learning. Synth. Lect. Artif. Intell. Mach. Learn. 2012, 6, 1-114.

(55) Podryabinkin, E. V.; Shapeev, A. V. Active Learning of Linearly Parametrized Interatomic Potentials. Comput. Mater. Sci. 2017, 140, 171-180.

(56) Smith, J. S.; Nebgen, B.; Lubbers, N.; Isayev, O.; Roitberg, A. E. Less Is More: Sampling Chemical Space with Active Learning. J. Chem. Phys. 2018, 148, 241733.

(57) Podryabinkin, E. V; Tikhonov, E. V; Shapeev, A. V; Oganov, A. R. Accelerating Crystal Structure Prediction by Machine-Learning Interatomic Potentials with Active Learning. Phys. Rev. B 2019, $99,64114$.

(58) Zhong, M.; Tran, K.; Min, Y.; Wang, C.; Wang, Z.; Dinh, C.-T.; De Luna, P.; Yu, Z.; Rasouli, A. S.; Brodersen, P.; Sun, S.; Voznyy, O.; Tan, C.-S.; Askerka, M.; Che, F.; Liu, M.; Seifitokaldani, A.; Pang, Y.; Lo, S.-C.; Ip, A.; Ulissi, Z.; Sargent, E. H. Accelerated Discovery of $\mathrm{CO}_{2}$ Electrocatalysts Using Active Machine Learning. Nature 2020, 581, 178-183.

(59) Coe, J. P. Machine Learning Configuration Interaction. J. Chem. Theory Comput. 2018, 14, 57395749.

(60) Coe, J. P. Machine Learning Configuration Interaction for Ab Initio Potential Energy Curves. $J$. Chem. Theory Comput. 2019, 15, 6179-6189. 
(61) Pineda Flores, S. D. Chembot: A Machine Learning Approach to Selective Configuration Interaction. J. Chem. Theory Comput. 2021. https://doi.org/10.1021/acs.jctc.1c00196.

(62) Ghosh, S. K.; Rano, M.; Ghosh, D. Configuration Interaction Trained by Neural Networks: Application to Model Polyaromatic Hydrocarbons. J. Chem. Phys. 2021, 154, 94117.

(63) Goings, J.; Hu, H.; Yang, C.; Li, X. Reinforcement Learning Configuration Interaction. 2021, chemrxiv.14342234.v2. https://doi.org/10.26434/chemrxiv.14342234.v2.

(64) Benkyi, I.; Tapavicza, E.; Fliegl, H.; Sundholm, D. Calculation of Vibrationally Resolved Absorption Spectra of Acenes and Pyrene. Phys. Chem. Chem. Phys. 2019, 21, 21094-21103.

(65) Yang, Y.; Davidson, E. R.; Yang, W. Nature of Ground and Electronic Excited States of Higher Acenes. Proc. Natl. Acad. Sci. 2016, 113, E5098-E5107.

(66) Bettinger, H. F.; Tönshoff, C.; Doerr, M.; Sanchez-Garcia, E. Electronically Excited States of Higher Acenes up to Nonacene: A Density Functional Theory/Multireference Configuration Interaction Study. J. Chem. Theory Comput. 2015, 12, 305-312.

(67) Ivanic, J.; Ruedenberg, K. Identification of Deadwood in Configuration Spaces through General Direct Configuration Interaction. Theor. Chem. Acc. 2001, 106, 339-351.

(68) Gordon, M. S.; Schmidt, M. W. Advances in Electronic Structure Theory: GAMESS a Decade Later. In Theory and applications of computational chemistry; Elsevier, 2005; pp 1167-1189.

(69) Schmidt, M. W.; Baldridge, K. K.; Boatz, J. A.; Elbert, S. T.; Gordon, M. S.; Jensen, J. H.; Koseki, S.; Matsunaga, N.; Nguyen, K. A.; Su, S. General Atomic and Molecular Electronic Structure System. J. Comput. Chem. 1993, 14, 1347-1363.

(70) Frisch, M. J.; Trucks, G. W.; Schlegel, H. B.; Scuseria, G. E.; Robb, M. A.; Cheeseman, J. R.; Scalmani, G.; Barone, V.; Mennucci, B.; Petersson, G. A. B Gaussian 09, Revision E. 01. Gaussian, Inc., Wallingford, CT, USA. 
(71) Zhao, Y.; Truhlar, D. G. A New Local Density Functional for Main-Group Thermochemistry, Transition Metal Bonding, Thermochemical Kinetics, and Noncovalent Interactions. J. Chem. Phys. 2006, 125, 194101.

(72) Weigend, F. Accurate Coulomb-Fitting Basis Sets for H to Rn. Phys. Chem. Chem. Phys. 2006, 8, 1057-1065.

(73) Weigend, F.; Ahlrichs, R. Balanced Basis Sets of Split Valence, Triple Zeta Valence and Quadruple Zeta Valence Quality for H to Rn: Design and Assessment of Accuracy. Phys. Chem. Chem. Phys. 2005, 7, 3297-3305.

(74) Dunning Jr, T. H. Gaussian Basis Sets for Use in Correlated Molecular Calculations. I. The Atoms Boron through Neon and Hydrogen. J. Chem. Phys. 1989, 90, 1007-1023.

(75) Murphy, K. P. Machine Learning: A Probabilistic Perspective; MIT press, 2012.

(76) Cunningham, P.; Delany, S. J. K-Nearest Neighbour Classifiers: 2nd Edition (with Python Examples). 2020. arXiv:2004.04523v2. arXiv.org e-Print archive. https://arxiv.org/abs/2004.04523

(77) Rasmussen, C. E. Gaussian Processes for Machine Learning; MIT Press, 2006.

(78) Breiman, L. Random Forests. Mach. Learn. 2001, 45, 5-32.

(79) Chen, T.; Guestrin, C. XGBoost: A Scalable Tree Boosting System. In Proceedings of the 22Nd ACM SIGKDD International Conference on Knowledge Discovery and Data Mining; KDD '16; ACM: New York, NY, USA, 2016; pp 785-794. https://doi.org/10.1145/2939672.2939785.

(80) Géron, A. Hands-on Machine Learning with Scikit-Learn, Keras, and TensorFlow: Concepts, Tools, and Techniques to Build Intelligent Systems; O’Reilly Media, 2019.

(81) Pedregosa, F.; Varoquaux, G.; Gramfort, A.; Michel, V.; Thirion, B.; Grisel, O.; Blondel, M.; 
Prettenhofer, P.; Weiss, R.; Dubourg, V. Scikit-Learn: Machine Learning in Python. J. Mach. Learn. Res. 2011, 12, 2825-2830.

(82) Tietz, M.; Fan, T. J.; Nouri, D.; Bossan, B.; skorch Developers. Skorch: A Scikit-Learn Compatible Neural Network Library That Wraps PyTorch. July 2017. https://skorch.readthedocs.io/en/stable/

(83) Paszke, A.; Gross, S.; Massa, F.; Lerer, A.; Bradbury, J.; Chanan, G.; Killeen, T.; Lin, Z.; Gimelshein, N.; Antiga, L.; Desmaison, A.; Kopf, A.; Yang, E.; DeVito, Z.; Raison, M.; Tejani, A.; Chilamkurthy, S.; Steiner, B.; Fang, L.; Bai, J.; Chintala, S. PyTorch: An Imperative Style, High-Performance Deep Learning Library. Adv. Neural Inf. Process. Syst. 2019, 8026-8037.

(84) Bergstra, J.; Komer, B.; Eliasmith, C.; Yamins, D.; Cox, D. D. Hyperopt: A Python Library for Model Selection and Hyperparameter Optimization. Comput. Sci. Discov. 2015, 8, 14008.

Plasser, F.; Lischka, H. Multi-Reference Configuration Interaction. Quantum Chemistry and Dynamics of Excited States. November 23, 2020, pp 277-297.

David Sherrill, C.; Schaefer, H. F. The Configuration Interaction Method: Advances in Highly Correlated Approaches; Löwdin, P.-O., Sabin, J. R., Zerner, M. C., Brändas, E. B. T.-A. in Q. C., Eds.; Academic Press, 1999; Vol. 34, pp 143-269.

Grimme, S.; Parac, M. Substantial Errors from Time-Dependent Density Functional Theory for the Calculation of Excited States of Large $\pi$ Systems. ChemPhysChem 2003, 4, 292-295.

(88) Lopata, K.; Reslan, R.; Kowalska, M.; Neuhauser, D.; Govind, N.; Kowalski, K. Excited-State Studies of Polyacenes: A Comparative Picture Using EOMCCSD, CR-EOMCCSD(T), RangeSeparated (LR/RT)-TDDFT, TD-PM3, and TD-ZINDO. J. Chem. Theory Comput. 2011, 7, 36863693.

(89) Andersson, K.; Malmqvist, P. Å.; Roos, B. O.; Sadlej, A. J.; Wolinski, K. Second-Order 
Perturbation Theory with a CASSCF Reference Function. J. Phys. Chem. 1990, 94, 5483-5488.

(90) Andersson, K.; Malmqvist, P. Å.; Roos, B. O. Second-Order Perturbation Theory with a Complete Active Space Self-Consistent Field Reference Function. J. Chem. Phys. 1992, 96, 1218-1226.

(91) Gagliardi, L.; Truhlar, D. G.; Li Manni, G.; Carlson, R. K.; Hoyer, C. E.; Bao, J. L. Multiconfiguration Pair-Density Functional Theory: A New Way To Treat Strongly Correlated Systems. Acc. Chem. Res. 2017, 50, 66-73.

(92) Abraham, V.; Mayhall, N. J. Selected Configuration Interaction in a Basis of Cluster State Tensor Products. J. Chem. Theory Comput. 2020, 16, 6098-6113.

(93) Fales, B. S.; Martínez, T. J. Efficient Treatment of Large Active Spaces through Multi-GPU Parallel Implementation of Direct Configuration Interaction. J. Chem. Theory Comput. 2020, 16, $1586-1596$.

(94) Wang, T.; Ma, Y.; Zhao, L.; Jiang, J. Portably Parallel Construction of a Configuration-interaction Wave Function from a Matrix-Product State Using the Charm++ Framework. J. Comput. Chem. 2020, 41, 2707-2721. 